\section{VULNERABLE CHILDREN AND THEIR CARE QUALITY ISSUES: A DESCRIPTIVE ANALYSIS OF A NATIONAL DATABASE}

Adhnan Omar, ${ }^{1}$ Philippa Rees, ${ }^{1}$ Huw Prosser Evans, ${ }^{1}$ Huw Williams, ${ }^{1}$ Alison Cooper, ${ }^{1}$ Sophie Banerjee, ${ }_{1}^{1}$ Peter Hibbert, ${ }^{2}$ Meredith Makeham, ${ }^{2}$ Gareth Parry, ${ }^{3}$ Liam Donaldson, ${ }^{4}$ Adrian Edwards, ${ }^{1}$ Andrew Carson-Stevens ${ }^{1}$. ${ }^{1}$ Cardiff University, United Kingdom-Wales; ${ }^{2}$ University of New South Wales, United Kingdom-Wales; ${ }^{3}$ Institute for Healthcare Improvement, United States; ${ }^{4}$ Imperial College London, United Kingdom-England

\subsection{6/bmjgs-2015-IHlabstracts. 18}

Background Globally, two in five children are deemed vulnerable by virtue of their physical, psychological and social characteristics. These children are at increased risk of suboptimal care and disproportionate levels of iatrogenic harm. There remains a paucity of literature describing initiatives for practice improvement for these children.

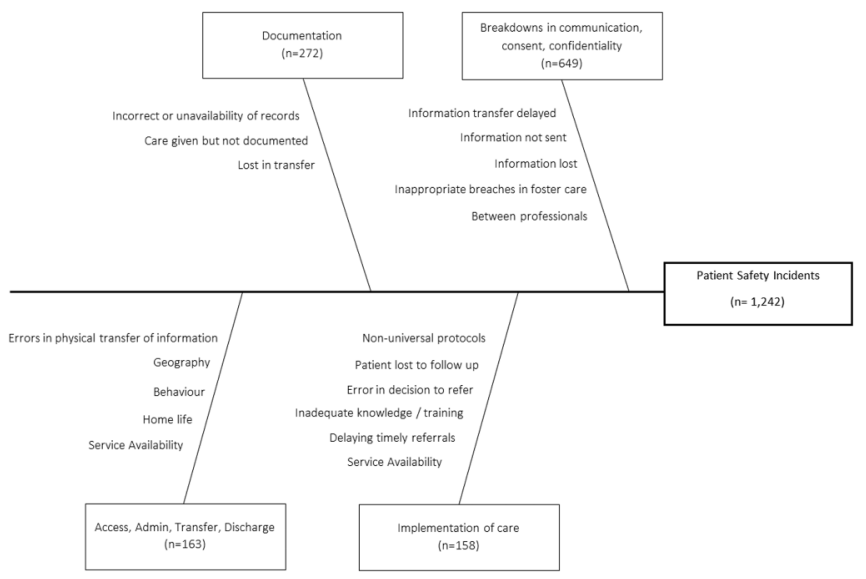

Figure 1 Ishikawa diagram illustrating causative factors contributing to patient safety incidents.

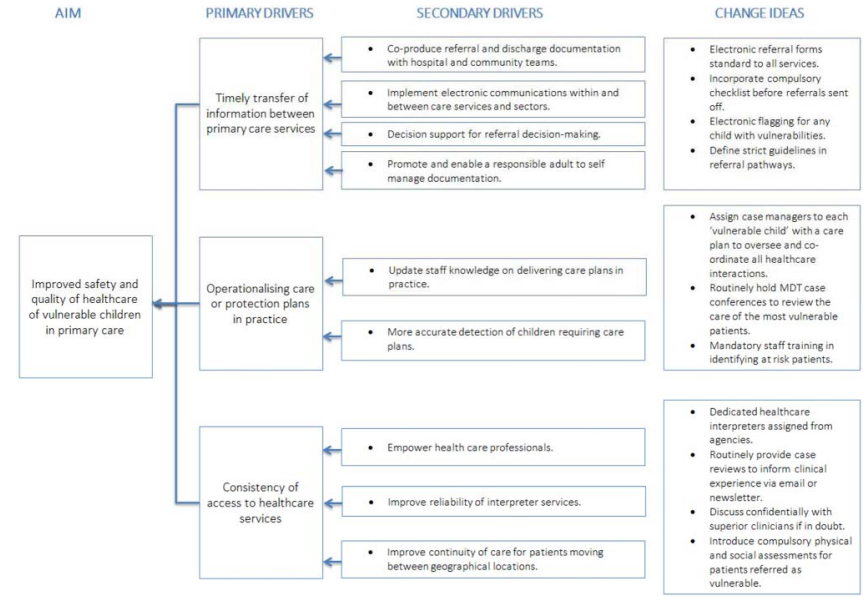

Figure 2 Driver diagram for improved safety and quality of healthcare of vulnerable children in primary care.

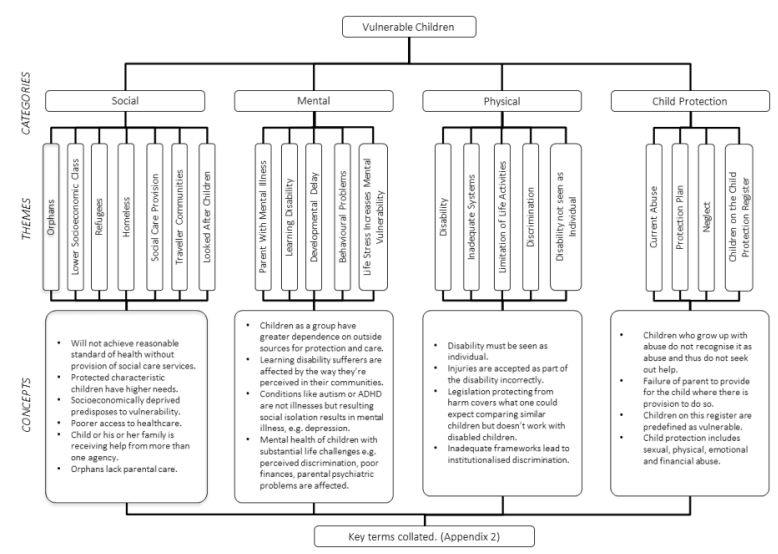

Appendix 1 Process of generating key terms.

Objectives Identify safety reports describing vulnerable children in the England and Wales National Reporting and Learning System (NRLS). Characterise reports in terms of what happened and reported causative issues, in order to identify key change concepts (drivers) and related interventions (change ideas) for improvement.

Methods An operational definition of 'vulnerability in children', including key domains and related keywords (Appendix

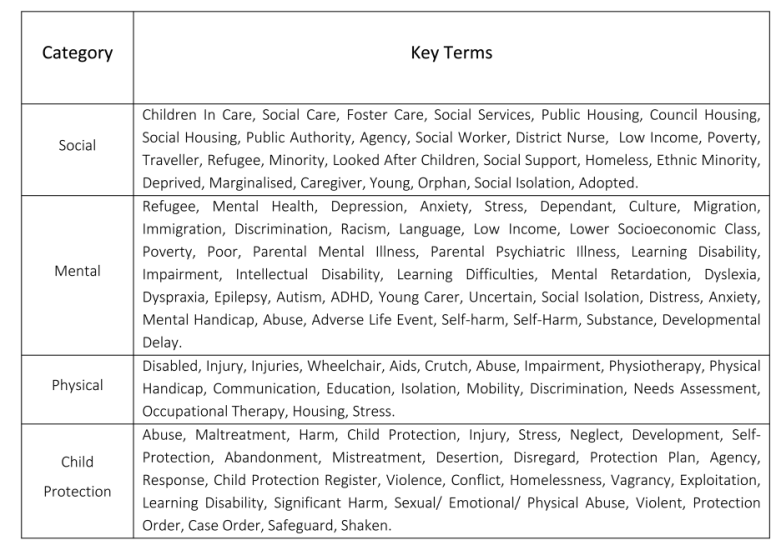

Appendix 2 Process of generating key terms (continued). 
1,2 ), was empirically informed by a scoping review of the published and grey literature. Relevant reports were descriptively analysed. The free text of the four most frequent incident types (Pareto principle) was discussed by a multi-disciplinary team to identify key concepts for improvement.

Results 2,015 reports were identified involving vulnerable children. The problem areas identified primarily resulted from a fragmentation of care services. This included inefficient transfer of information between primary care services; failure to operationalise care plans in practice; and inconsistent access to healthcare services. Reported causes are outlined in an Ishikawa diagram (figure 1) and summarized in the related driver diagram (figure 2) to mitigate problem areas.

Conclusions Analysis of patient safety incident reports assisted in the pragmatic identification of key concepts for healthcare professionals to begin more informed discussions about improving the care delivered to vulnerable children. 